\title{
Comodulation Masking Release In Electric Hearing
}

\author{
Robert H. Pierzycki ${ }^{1,2}$ and Bernhard U. Seeber ${ }^{1,3}$ \\ ${ }^{1}$ MRC Institute of Hearing Research, University Park, Nottingham, NG7 2RD, UK \\ ${ }^{2}$ Present Address: NIHR Nottingham Hearing Biomedical Research Unit, Ropewalk House, 113 The Ropewalk, Nottingham, \\ NG1 5DU, UK \\ ${ }^{3}$ Present Address: Audio Information Processing, Department of Electrical Engineering and Information Technology, Technische \\ Universität München, Arcisstrasse 21, 80333 Munich, Germany
}

Received: 15 March 2011; Accepted: 16 December 2013; Online publication: 11 January 2014

\begin{abstract}
Comodulation masking release (CMR) is an improvement in the detection threshold of a masked signal that occurs when the masker envelopes are correlated across frequency (i.e., comodulation). CMR can be observed when flanking bands (FBs) of noise comodulated with an on-frequency band (OFB) noise masker are added at remote frequencies (CMR1), or when co-modulated envelopes are used instead of anti-modulated envelopes (OFB and FB envelopes out of phase, CMR2). For FBs widely separated from the $\mathrm{OFB}$, this process is assumed to rely mostly on acrosschannel comparison of temporal envelopes. Since cochlear implants (CIs) rely predominantly on the transmission of envelope cues, we investigated if CMR can be observed in electric hearing. We stimulated the auditory nerve of eight CI users with trains of modulated electric pulses presented on an OFB electrode alone, or together with pulse trains on one or two FB electrodes. Participants had to detect signalinduced changes in the envelope of an electric pulse train masker presented on the OFB electrode. Envelopes on FB electrodes were either co-modulated or anti-modulated with the envelope of the OFB masker. We observed CMR1 in one of the eight CI users. However, significant CMR2 was observed in most CI users. Reducing amplitude-modulation rate from 20 to $8 \mathrm{~Hz}$, reducing envelopes' randomness or increas-
\end{abstract}

Correspondence to: Robert H. Pierzycki • NIHR Nottingham Hearing Biomedical Research Unit, Ropewalk House • 113 The Ropewalk, Nottingham NG1 5DU, UK. Telephone: +44-115-8232829 fax: +44-115-8232618 email: rpierzycki@gmail.com ing electrode separation did not generally improve CMR1, but increased the prevalence of CMR2. The present results suggest that comodulation of envelopes can aid signal detection in electric hearing.

Keywords: cochlear implant, temporal processing, signal detection, envelope, temporal fine structure, electrical stimulation

\section{INTRODUCTION}

In normal hearing $(\mathrm{NH})$ listeners, detection of a signal masked by an on-frequency noise band (OFB) masker can be improved by presenting flanking bands (FBs) of noise at frequencies remote from the OFB. This release from masking requires coherent envelope modulations in the OFB and FBs and it is thus termed comodulation masking release (CMR) (Hall et al. 1984).

A number of studies have shown that CMR can be partly explained by processing within the OFB channel alone-the within-channel CMR (McFadden 1986; Schooneveldt and Moore 1987; Verhey et al. 1999). This effect is related to the detection of changes in the envelope resulting from beating between the OFB and FBs within the target channel, especially for FBs positioned relatively close to the OFB (Goldman et al. 2010). However, CMR can be obtained with FBs as far as three octaves away from the OFB (Cohen 1991; Ernst and Verhey 2005), suggesting that an acrosschannel process which relies on the comparison of temporal envelopes at the outputs of separate audito- 
ry filters contributes to CMR. The across-channel mechanism is often associated with dip-listening where the comparison of dips in the OFB and FBs gives a cue for when to listen for the signal in the target channel (Buus 1985). Alternative models suggest that CMR may rely on the equalization-cancelation mechanism where equalization and subtraction of the FB and OFB envelopes maximizes the SNR in the OFB channel (Buus 1985), or on the detection of the reduced correlation between the OFB and FB envelopes due to the presence of the signal (Richards 1987).

In hearing with cochlear implants (CIs), or electric hearing, multiple electrodes inserted into the cochlea stimulate the auditory nerve with fast-rate electric pulse trains. The pulse trains are modulated with envelopes extracted from incoming sounds by the CI processor. Centrally mediated across-channel temporal processing has been shown to occur in electric hearing, evidenced by reduced sensitivity to changes of signal modulation after introducing modulated maskers on remote electrodes (Chatterjee 2003; Chatterjee and Oba 2004), or by the ability to detect small across-channel timing differences (Carlyon et al. 2000). Thus, processing across CI electrodes may also give rise to $\mathrm{CMR}$.

Wagner (2002) investigated CMR in individuals using a CI and their own speech processor, and observed a small CMR (about 2.6 dB). The OFB and the FB noise bands were presented via loudspeakers and were designed to fall within a single bandpass filter of the CI processor. Thus CMR was based on within-channel cues. A more recent study investigated speech perception in noise under conditions of CMR with CIs (Ihlefeld et al. 2012). No benefit to speech understanding from comodulation was found. The absence of CMR might have been due to the fact that speech identification requires a more detailed stimulus analysis than a detection task. A recent study using direct electric stimulation of CIs with a clinical coding strategy has shown CMR in about a third of tested CI users (Zirn et al. 2013). However, although it was argued that etiology of deafness gives some prediction for the presence or absence of CMR in CI users, it is still not entirely clear what are the main factors or mechanisms affecting CMR in electric hearing.

Our previous CMR experiments with $\mathrm{NH}$ listeners showed significant CMR with vocoded stimuli (Pierzycki and Seeber 2010). Vocoding simulates CI processing by replacing the temporal fine structure (TFS) interactions between signal and masker with unrelated TFS (noise or sinusoid carriers) while largely maintaining the stimulus envelopes. That significant CMR was observed using vocoding and led us to conclude that CMR is a robust process relying mainly on envelope cues. We therefore hypothesized that CMR could be observed even in CI users whose CI processors replace the input signals' TFS information with constant-rate electric pulse trains.

In the present study, we tested if co-modulated pulse trains delivered directly via a research interface to separate OFB and FB electrodes, thereby bypassing the user's CI processor, can improve signal detection and lead to CMR in electric hearing. We tested a number of factors and mechanisms that are known to be related to CMR, and used two ways of measuring CMR to extensively investigate CMR in electric hearing. The electric pulse trains were amplitudemodulated in one of two ways: (a) with the envelopes extracted from narrow-band noises with the addition of a low-rate sinusoidal amplitude modulation (SAM), (b) directly with a low-rate SAM. In experiment 1 , we tested CMR with the stimuli used in our vocoder study with $\mathrm{NH}$ listeners to test the hypothesis that CMR occurs in electric hearing as suggested by the results of that previous study. To investigate the effects of stimulus parameters on dip-listening, we studied the effect of decreased SAM rate on CMR in experiment 2 , and the effect of controlling masking from envelope peaks into masker dips in experiment 3 by using deterministic envelopes. Finally, in experiment 4, we tested the effect of current spread on CMR in electric hearing by increasing the OFB-FB separation.

\section{METHODS}

\section{Participants}

Eight post-lingually deafened adult CI users took part in the experiments. All were implanted with an Advanced Bionics CI HiRes90k implant, apart from participant $\mathrm{AB} 04$ who was implanted with the CII implant, and had more than 1 year of experience with CIs. Detailed participant information is shown in Table 1. All participants were paid for participation. The study was approved by the National Research Ethics Committee (UK).

\section{Definition of CMR and Overview of Conditions}

Three conditions were tested in each experiment following the paradigm of our study with $\mathrm{NH}$ listeners (Pierzycki and Seeber 2010). In the OFB-only condition, the signal threshold was measured when stimulating only on the selected OFB electrode. In the two other conditions, in addition to stimulation on the OFB electrode, one or two FB electrodes were stimulated with co-modulated or anti-modulated pulse trains (i.e., FB envelopes in phase or out-of-phase with OFB envelope), referred to as the CM or AntiM conditions, respectively. In experiments 1, 2, and 3, 
TABLE 1

Details of $\mathrm{Cl}$ users who participated in the experiments

\begin{tabular}{|c|c|c|c|c|}
\hline Subject & Age (years) & Etiology & $\begin{array}{l}\text { Duration of hearing } \\
\text { impairment before } \\
\text { implantation/implant } \\
\text { use (years) }\end{array}$ & $\begin{array}{l}\text { Chosen electrodes } \\
\text { apical FB-OFB- } \\
\text { basal FB }\end{array}$ \\
\hline AB01 & 62 & Unknown & $7 / 4$ & $4-9-14$ \\
\hline ABO2 & 66 & Ménière's Disease & $6.5 / 5$ & $4-8-12$ \\
\hline AB03 & 66 & Noise exposure & $8 / 3$ & $4-9-14$ \\
\hline AB04 & 60 & Measles & $45 / 8$ & $4-9-14$ \\
\hline AB05 & 62 & Hereditary & $20 / 2$ & $4-9-14$ \\
\hline AB06 & 70 & Injury & $62.5 / 5$ & $4-9-14$ \\
\hline AB07 & 64 & Otosclerosis & $48 / 5$ & $4-9-14$ \\
\hline AB08 & 59 & Unknown & $25 / 3$ & $4-9-14$ \\
\hline
\end{tabular}

FBs were delivered to selected apical and basal FB electrodes located symmetrically around the mid-array OFB electrode (Table 1). In experiment 4 , to test the effect of increasing electrode separation, OFB was delivered to the basal electrode and a single FB to the apical electrode. Furthermore, to maximize envelope correlation in experiment 4 , the OFB's envelope was also used to modulate the pulse trains on the FB electrode in the CM condition, but the AntiM envelope was extracted from a different noise sample.

CMR1 occurred when the CM thresholds were less than the OFB-only thresholds, i.e., thresholds were reduced when $\mathrm{CM}$ stimulation was added on the $\mathrm{FB}$ electrodes. CMR2 occurred when the CM thresholds were lower than the AntiM thresholds (Pierzycki and Seeber 2010). The CMR2 definition has the advantage that the envelope spectra are similar across the electrode array and that the same electrodes are stimulated in both conditions. CMR2 is usually larger than CMR1 (Schooneveldt and Moore 1987; Verhey et al. 2003).

\section{Stimuli and Equipment}

Stimuli used in experiments 1, 2, and 4 will be referred to as "acoustic", because the constant-rate electric pulse trains were modulated with envelopes extracted from CMR stimuli generated in a similar way as in our experiments with NH listeners, i.e., in acoustic hearing (for detailed description see Pierzycki and Seeber 2010). The samples of narrowband $\mathrm{OFB}$ and $\mathrm{FB}$ noises and a tonal signal were generated digitally on each trial. The OFB and FB noises were 500-ms-long and $60 \mathrm{~dB}$ SPL before applying SAM. The center frequencies of the one ERB-wide noise bands were (bandwidths in brackets): $443 \mathrm{~Hz}(72 \mathrm{~Hz})$ for apical FB, $1,053 \mathrm{~Hz}(138 \mathrm{~Hz})$ for the $\mathrm{OFB}$, and $2,216 \mathrm{~Hz}(263 \mathrm{~Hz})$ for the basal $\mathrm{FB}$ (Pierzycki and Seeber 2010). The SAM rate was $20 \mathrm{~Hz}$ (experiment 1) or $8 \mathrm{~Hz}$ (experiments 2-4) which resulted in ten or four modulation periods, respec- tively. The modulation depth was $100 \%$ and a $-\pi / 2$ and $+\pi / 2$ modulation phase was used for the $\mathrm{CM}$ and AntiM FBs, respectively. The noise bands and the signal had 20-ms-long Gaussian ramps applied before being summed into a combined masker or a masker plus signal stimulus. The 240-ms-long tonal signal of $1,053 \mathrm{~Hz}$ was positioned such that the temporal center of its onset and offset ramps coincided approximately with the SAM minimum in the masker. Signal level was varied using an adaptive tracking procedure.

The subsequent processing of the acoustic stimuli imitated that of continuous interleaved sampling strategies frequently used with CIs (Wilson et al. 1991). The envelopes of the OFB and FB noise bands and of the OFB noise with the added tone were extracted by half-wave rectification and low-pass filtering at $200 \mathrm{~Hz}$. Extracted envelopes were normalized such that the highest peak across the OFB and FB envelopes was equal to one. Next, the logarithm of the envelope amplitudes was taken (conversion to $\mathrm{dB}$ ) and mapped to an electric current in microamperes. All levels between the peak of the normalized envelope and a level $30 \mathrm{~dB}$ below the peak were mapped linearly between the participant's loudnessbalanced current $(\mathrm{Ib})$ and the threshold $(\mathrm{T})$ level on each corresponding electrode (see loudness balancing and $\mathrm{T}$ level procedures below, Fig. 2). This normalization ensured that the peaks of the OFB and FB envelopes never exceeded the loudness-balanced currents. Envelope samples at levels lower than $30 \mathrm{~dB}$ below the peak were linearly extrapolated to positive current units below the $\mathrm{T}$ level using the same mapping slope. If the addition of the signal to the OFB resulted in SPLs higher than $60 \mathrm{~dB}$ (the level of the unmodulated noise carrier for the OFB), levels were mapped linearly to currents higher than $\mathrm{Ib}$ on the OFB electrode, but limited to the participant's most comfortable (M) level on that electrode (see M level procedure below). The electric pulse train carriers were then modulated with the mapped 


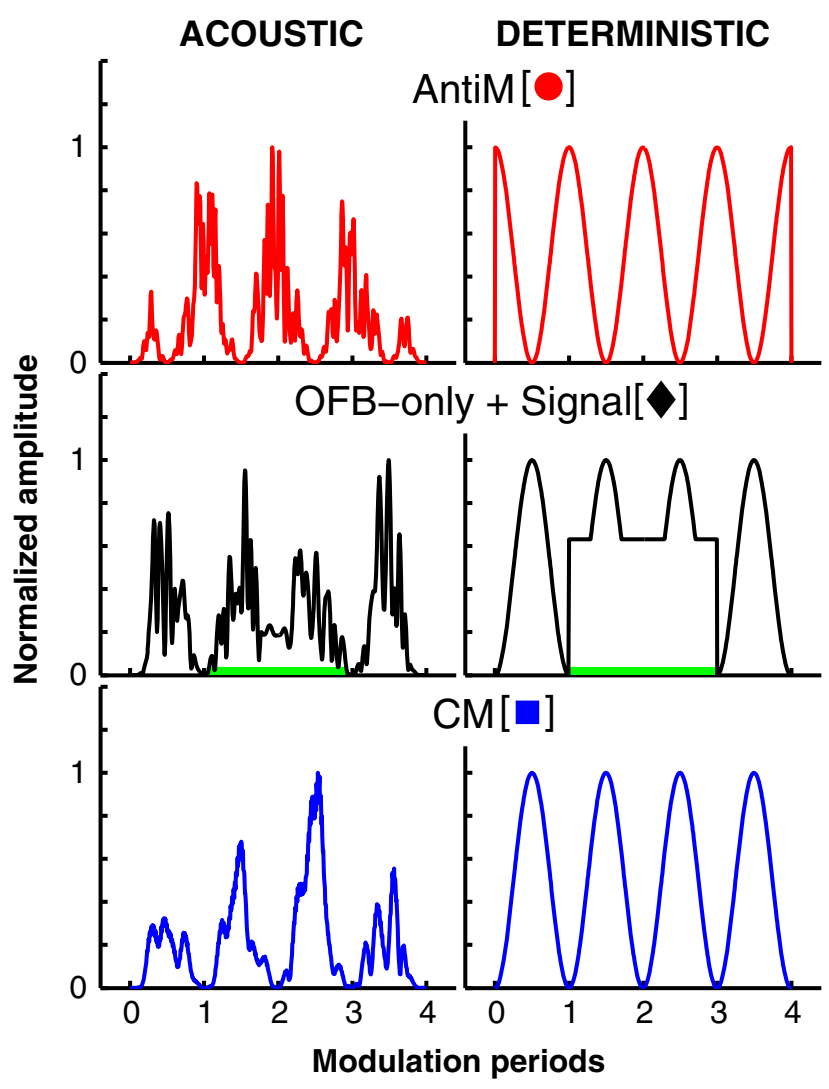

FIG. 1. Example stimuli envelopes (8 Hz SAM) used to modulate electric pulse trains in experiments with acoustic (left) and deterministic envelopes (right). The rows represent envelopes of the antimodulated flanker, OFB with added signal, and co-modulated FB (top to bottom). The signal level was set to the across-participant average OFB-only threshold in corresponding conditions. The green line in the middle row indicates the temporal position of the signal (NB the "filling in" of the second OFB envelope minimum due to the addition of the signal). The symbols in brackets are used for corresponding thresholds in Figures 3, 4, and 5.

envelopes. A schematic of stimuli envelopes is shown in Figure 1.

In experiment 3 , we used stimuli with deterministic envelopes generated by direct SAM of 500-ms-long electric pulse trains at $8 \mathrm{~Hz}$. This resulted in idealized, sinusoidal envelope fluctuations and maximum envelope correlation across electrodes. The modulation depth was $100 \%$, i.e., between $0 \mu \mathrm{A}$ and the loudnessbalanced current, Ib (Fig. 2). The phase of the SAM was the same as in the acoustic stimuli, but no Gaussian ramps were applied to the pulse trains' onsets/offsets. The signal was represented by setting the 240-ms-long center portion of the OFB pulse train to a current value determined on each trial by the adaptive tracking procedure. For signal currents higher than the peak amplitude of the SAM, the signal was represented by constant-amplitude pulses (flat envelope), whereas for signal currents lower than the peak amplitude of the SAM it was represented by "filling in" the SAM minima (Fig. 1). The signal's onset and offset were in the temporal center of the first and last minimum of the OFB pulse train, respectively. No ramps were applied to the signal to provide a steep onset cue against the SAM of the OFB masker.

In all experiments, bi-phasic electric pulse trains were used as carriers that stimulated three electrodes in monopolar mode. The phase duration of the electric pulses was $40 \mu$ s with a $20-\mu$ s-long interphase gap. A 1,205-pps carrier rate was used on each electrode. To minimize channel interactions, electrodes were stimulated in a "staggered" order: the first bi-phasic pulse was presented on the midarray electrode followed by the pulses on the apical and the basal electrodes with equal periods between the pulses (approx. $177 \mu$ s). Stimuli were presented via an Advanced Bionics research interface (HRStream 1.01) and a blank Platinum Sound Processor with headpiece.

Prior to the experiments, the impedance of each electrode of the participant's implant was measured in the Advanced Bionics SoundWave package (Version 1.4.77). Electrodes with relatively low impedance were selected (Table 1).

\section{Procedures}

$\mathbf{M}$ and $\mathbf{T}$ Levels. The threshold $(\mathrm{T})$ and most comfortable level (M) were measured at selected electrodes using 500 ms-long pulse trains with SAM at $20 \mathrm{~Hz}$ (pilot measurements showed almost no change in $\mathrm{T}$ and $\mathrm{M}$ levels when the modulation rate was reduced from 20 to $8 \mathrm{~Hz}$ ). An adjustment procedure was used for the $\mathrm{M}$ level measurement in which participants increased or decreased the stimulus current by pressing buttons associated with "small", "medium", or "large" current steps (5, 10, or $20 \mu \mathrm{A}$, respectively). Participants were asked to use the smaller step sizes once the sound was clearly audible. As a safety precaution, a stimulation limit of $500 \mu \mathrm{A}$ was implemented in the procedure's code. If the limit was reached, three consecutive button presses asking to increase the level were required before the current limit was raised by $100 \mu \mathrm{A}$. When this new limit was reached, it could be increased by a further $100 \mu \mathrm{A}$ only with three consecutive button presses, and so on. When the stimulus was perceived as most comfortable, the participant stopped the run by pressing the "accept" button and the $\mathrm{M}$ level was stored. The $\mathrm{M}$ levels were measured twice for each electrode and the rounded mean of the two measurements was used.

The T level was measured using a two-interval forcedchoice adaptive procedure with one interval being silent $(0 \mu \mathrm{A}$ pulse train) and one carrying the signal (pulse 


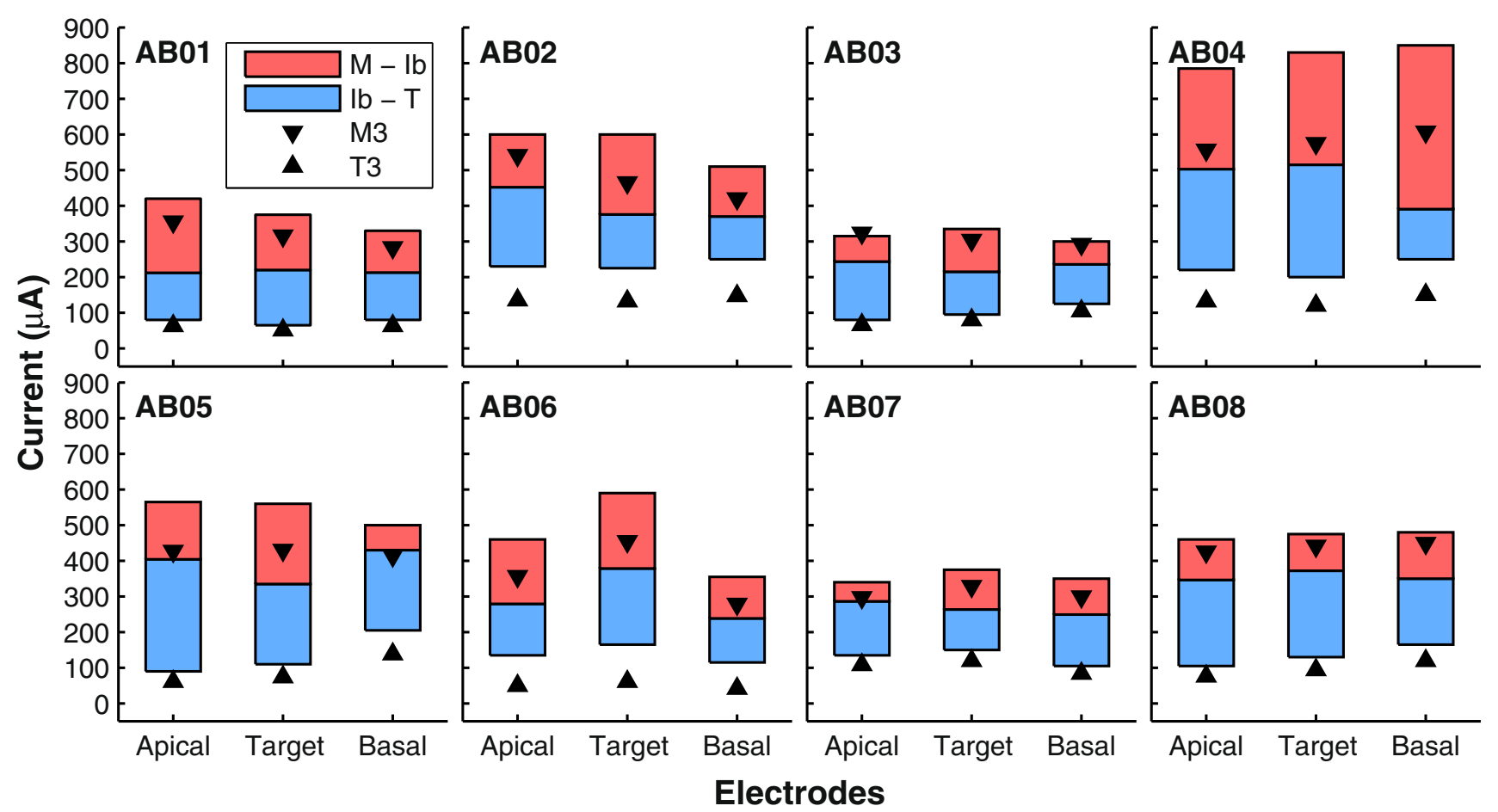

FIG. 2. Participants' most comfortable and threshold levels measured on single electrodes ( $\mathrm{M}, \mathrm{T}$; boxes) and for stimulation on all three electrodes (M3 downward triangles, T3 upward triangles). The height of each box extends from the $\mathrm{T}$ to $\mathrm{M}$ level and thus

train with SAM as in the M level measurements). The inter-stimulus interval was $300 \mathrm{~ms}$. The intervals were visually cued by highlighting one of the two response buttons displayed on a computer screen. Participants were asked to indicate the interval that contained the sound. Visual feedback was given after each response. The initial stimulus current of $75 \%$ of the $\mathrm{M}$ level was changed in a 2-down 1-up adaptive tracking procedure (Levitt 1971) with current steps changing from an initial $20 \mu \mathrm{A}$ to 10 to $5 \mu \mathrm{A}$ after the first and second reversal, respectively. Six reversals were tracked at the smallest current step and their average was stored as the T level for this electrode. One track was measured for each $\mathrm{T}$ level.

The $\mathrm{T}$ and $\mathrm{M}$ levels were also measured by stimulating all three electrodes (OFB and 2 FBs) to assess the loudness summation. The levels will be referred to as the T3 and M3. The procedure was essentially the same as in the single electrode measurements and pulse trains with applied SAM were used. However, because $\mathrm{T}$ and $\mathrm{M}$ levels differed between electrodes the current was changed in equal steps on an electrode-specific scale taking the $\mathrm{T}$ and $\mathrm{M}$ levels into account. The scale was computed for each electrode by linearly interpolating 201 points between 0 , $\mathrm{T}$, and $\mathrm{M}$ levels (all in microamperes), i.e., 0-100-200 in the $0-\mathrm{T}-\mathrm{M}$ range. In the M3 measurement the current was increased or decreased by the represents the electric dynamic range (EDR) on a particular electrode. The horizontal line splitting each box into blue and red shaded areas depicts the loudness-balanced current (lb).

participant in small, medium, and large steps of 2, 5, and 10 points, respectively, on the derived current scale. In the T3 measurement, the steps of the adaptive track were set to 10,5 , and 2 points on the current scale. The M3 was taken as the mean of two runs and a single run was taken for T3. The T, M, T3, and M3 levels and the Electric Dynamic Range (EDR= $\mathrm{M}-\mathrm{T})$ for each participant are shown in Figure 2.

Loudness Balancing. A loudness balancing procedure was used to match the loudness of the electric pulse trains on the FB electrodes to that on the OFB electrode. A 500-ms-long carrier pulse train with $20 \mathrm{~Hz}$ SAM was presented in two intervals with equal probabilities on the $\mathrm{OFB}$ and on one of the FB electrodes. The inter-stimulus interval was $300 \mathrm{~ms}$. Participants indicated which interval contained the louder sound. The current of the pulse train on the OFB electrode was fixed to $50 \%$ of the EDR on that electrode, whereas the current on the FB electrode was changed according to an adaptive procedure. Four loudness-balanced current (Ib) estimates were obtained for each FB electrode. Two estimates were obtained from 2-down 1-up adaptive tracks in which the current on the FB electrode was initially descending from the level of $80 \%$ of the EDR on that electrode. Two other estimates were obtained from 2-up 1-down adaptive tracks with the current initially ascending from $30 \%$ of the EDR. The current 
was tracked in microamperes and the first and second reversal reduced the current step size from an initial $10 \mu \mathrm{A}$ to 5 to $2 \mu \mathrm{A}$. The Ib for each track was stored as the mean of six reversals at the smallest current step. The participant's final $\mathrm{Ib}$ was taken as the mean across the four current estimates (Fig. 2).

The Ib on the OFB electrode was typically chosen as $50 \%$ of its EDR which was usually lower than M3 on the OFB electrode. Thus, current summation from stimulating three electrodes resulted in lower loudness than "most comfortable loudness" (M3 level) and allowed headroom for the presence of the signal. However, participant AB02 showed large current summation in that M3 was lower than $50 \%$ EDR on the OFB electrode. For this participant Ib was reduced to $40 \%$ EDR on the OFB electrode, and scaled down accordingly on FB electrodes to maintain the loudness balance. The stimuli mapped to the measured loudness-balanced currents were too quiet for participant $\mathrm{AB} 08$. Therefore, for $\mathrm{AB} 08$, the $\mathrm{Ib}$ on the target electrode was increased to $70 \%$ of its EDR and the Ib on FB electrodes was scaled accordingly.

CMR Experiments. In the CMR experiments signal thresholds were measured in a two-alternative forcedchoice procedure with each trial consisting of three intervals separated by a $400 \mathrm{~ms}$ inter-stimulus interval. The first interval always contained the anchor sound, the masker alone (OFB-only or the OFB with $\mathrm{CM}$ or AntiM FBs), while the second and third intervals contained the masker alone or the masker plus signal with equal probabilities. Participants indicated which interval was different from the anchor. Visual feedback was given after every response.

The signal level was tracked using a 3-down 1-up adaptive procedure estimating the $79.4 \%$ correct point on the psychometric function (Levitt 1971). In experiments 1, 2, and 4 with acoustic envelopes, the signal level was tracked in dB SPL (i.e., before mapping to electric current) from an initial $70 \mathrm{~dB}$ SPL. The level was initially reduced with a step size of $6 \mathrm{~dB}$, and was tracked with step sizes of 6,4 , and $2 \mathrm{~dB}$ after the first, second, and third reversal, respectively. The level was then kept at $2 \mathrm{~dB}$ and tracked for a further six reversals. The tracks were terminated after the ninth reversal. Three tracks were obtained for each condition (OFB-only, CM, and AntiM), and the conditions were run in a random order. In experiment 3, with deterministic envelopes, the adaptive tracking of the signal's current was done in percentage steps of EDR on the OFB electrode. The initial signal level was set at $20 \%$ above the masker level on the OFB electrode, i.e., at $70 \%$ EDR for all but AB02 and $\mathrm{AB} 08$ whose initial signal levels were 60 and $90 \%$ EDR, respectively. The 3-down 1-up tracking procedure was the same as in experiments 1,2 , and 4, but the steps were $10,10,5$, and $2 \%$ of the EDR. Each track was terminated after the ninth reversal. Five tracks were run for each condition.

\section{Data Analysis}

We used the levels of last six reversals pooled across all runs to calculate individual thresholds and $95 \%$ confidence intervals using a bootstrapping technique. The bootstrapping technique allows estimating the sampling distribution of a statistic, in our case mean, by repeatedly sampling with replacement from the original data set a large number of times (Efron and Tibshirani 1993). The percentile method can then be used to calculate the limits of confidence intervals from the estimated sampling distribution.

For calculation of mean thresholds and corresponding $95 \%$ confidence intervals we resampled the final reversals $10^{6}$ times with a constraint that sampled reversals follow the "up-down" order as in the experimental adaptive tracks. The group thresholds with corresponding $95 \%$ confidence intervals were calculated by bootstrapping the $N$ individual bootstrapped thresholds $10^{6}$ times, where $N$ is the number of participants.

Individual CMR results and $95 \%$ confidence intervals were calculated from the difference between individual bootstrap thresholds for corresponding conditions, e.g., OFB-only - CM for CMR1. The group CMR results were obtained by bootstrapping across $N$ threshold differences from original data set to ensure that corresponding thresholds, e.g., OFB and CM for CMR1, came from the same participant before bootstrapping. Significant group CMR was reported when the $95 \%$ confidence intervals did not contain zero (i.e., significant at alpha $=0.05$ ). If CMR did not reach significance at a group level, we used the Bonferroni correction for the significance level, i.e., alpha $=0.05 / N$, and calculated new confidence intervals for individual CMR e.g. for $N=8$ alpha is approximately equal to 0.006 and $99.4 \%$ confidence intervals were calculated from sampling distributions. In this case, the participant's CMR was significant when the $99.4 \%$ confidence interval did not contain zero.

Note that although some authors adopted the use of a "negative CMR" term (Ernst et al. 2010), in the present study, only CMR values greater than zero indicate a release from masking and thus CMR. CMR values less than zero indicate increase in thresholds and thus more masking.

All figures show bootstrap thresholds as signal-tonoise ratios (SNR, decibels) and $95 \%$ confidence intervals. The thresholds were converted to SNRs using the signal's threshold level and the unmodulated OFB level, i.e., $60 \mathrm{~dB}$ SPL or $\mathrm{Ib}$ on the OFB 
electrode for the acoustic or deterministic stimuli, respectively.

\section{RESULTS}

\section{Experiment 1: Effect of Comodulation on Signal Detection}

In a previous study, $\mathrm{NH}$ listeners demonstrated CMR when listening to vocoded stimuli, suggesting that CMR is possible with cues restricted to the envelope (Pierzycki and Seeber 2010). In experiment 1, the paradigm and stimuli used in that study were adapted to test whether CI users demonstrate CMR. Using direct electric stimulation of the auditory nerve we tested whether signal detection is improved for comodulated pulse trains presented on separate CI electrodes. Panels in the left column of Figure 3 show individual signal-to-noise ratios (SNRs) for detecting the signal and associated $95 \%$ confidence intervals for each condition. The bottom panel presents group results.

CMR1 was not significant on a group level. Results of individual CI users showed significant CMR1 after Bonferroni correction for participant AB08 for whom the threshold with co-modulated FBs was $3.4 \mathrm{~dB}$ lower than for the OFB presented alone (Table 2). A trend toward lower CM than AntiM thresholds, indicating CMR2, was observed for most participants. CMR2 reached 2-3 dB and was significant for $\mathrm{AB} 02, \mathrm{AB} 06$, and $\mathrm{AB} 08$. This is also reflected on a group level where CMR2 was $1.1 \mathrm{~dB}$ and significant, but CMR1 was not. The across-participant SNR in the OFB-only condition was $-7.4 \mathrm{~dB}$, and $-7.1 \mathrm{~dB}$ and $-6.0 \mathrm{~dB}$ for the $\mathrm{CM}$ and AntiM conditions, respectively (Table 2).

In the present experiment signal thresholds were on average $7.4 \mathrm{~dB}$ lower than the $60 \mathrm{~dB}$ level of the OFB noise at its maximum (prior to SAM) which is equivalent to about $40 \%$ effective modulation depth. This suggests that our CI users could only take a moderate advantage of the $100 \%$-deep dips in the masker's SAM to listen for the signal. A possible reason is that forward masking on the OFB electrode filled the dips in the masker and prevented CMR. Therefore, in experiment 2, we reduced the modulation rate of the SAM to $8 \mathrm{~Hz}$, giving more than a twofold increase in the duration of the SAM dips in

FIG. 3. Signal thresholds expressed as signal-to-noise ratios (SNR, $\mathrm{dB}$ re-unmodulated masker level of $60 \mathrm{~dB}$ SPL; c.f. rms level of SAM masker was about $55 \mathrm{~dB}$ SPL) as a function of the number of FBs in the OFB-only (diamonds), anti-modulated (AntiM, circles) and comodulated (CM, squares) conditions. The left and right columns show the data for SAM rates of 20 and $8 \mathrm{~Hz}$, respectively. Results are shown as mean thresholds with $95 \%$ confidence intervals. The top eight rows represent different participants and the bottom row shows the group mean with $95 \%$ confidence intervals.

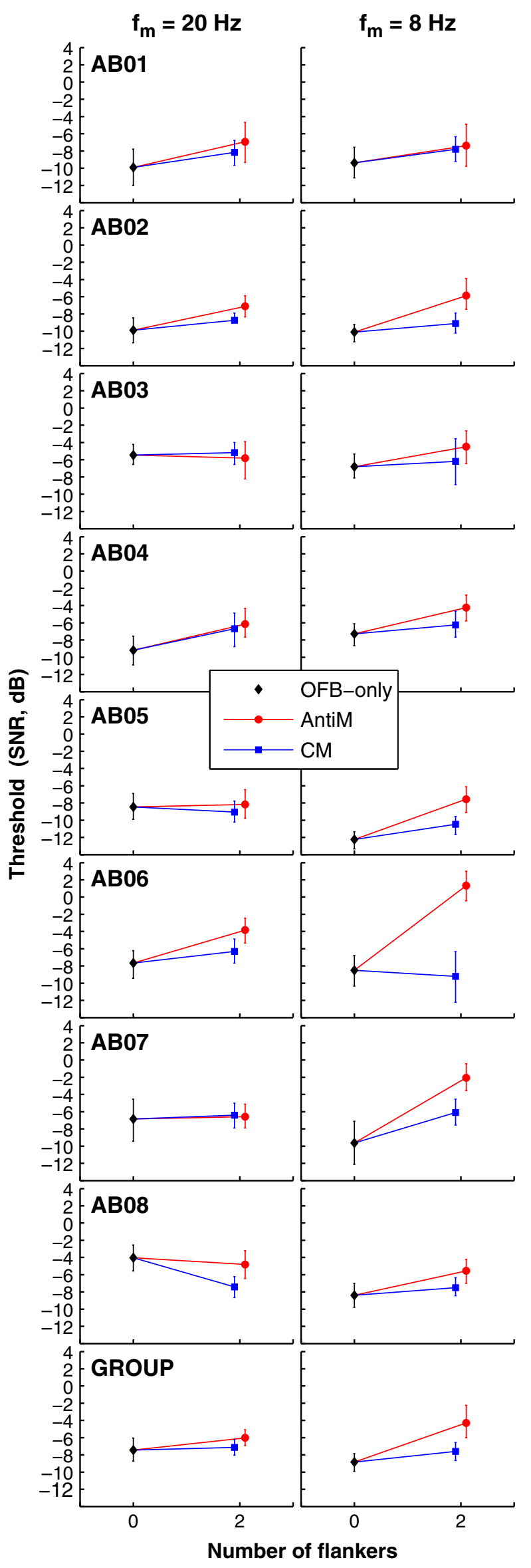


TABLE 2

\begin{tabular}{|c|c|c|c|c|c|c|}
\hline \multicolumn{7}{|c|}{ Results of CMR experiments } \\
\hline \multirow[b]{2}{*}{ Experiment } & \multirow[b]{2}{*}{ Subject } & \multicolumn{3}{|c|}{ Thresholds (dB SNR) } & \multirow{2}{*}{$\begin{array}{l}\text { CMR1 (dB) } \\
\text { OFB-CM }\end{array}$} & \multirow{2}{*}{$\begin{array}{l}C M R 2(d B) \\
\text { AntiM-CM }\end{array}$} \\
\hline & & OFB & $C M$ & AntiM & & \\
\hline \multirow[t]{9}{*}{ Experiment $1: 2 \mathrm{FBs}, f_{\mathrm{m}}=20 \mathrm{~Hz}$, acoustic envelopes } & AB01 & -9.9 & -8.2 & -6.9 & -1.7 & 1.3 \\
\hline & $\mathrm{ABO} 2$ & -9.9 & -8.7 & -7.1 & -1.1 & $1.6^{\mathrm{a}}$ \\
\hline & AB03 & -5.5 & -5.2 & -5.8 & -0.3 & -0.6 \\
\hline & $\mathrm{ABO} 4$ & -9.2 & -6.7 & -6.2 & -2.5 & 0.5 \\
\hline & AB05 & -8.5 & -9.1 & -8.2 & 0.6 & 0.9 \\
\hline & AB06 & -7.7 & -6.3 & -3.8 & -1.3 & $2.5^{\mathrm{a}}$ \\
\hline & $\mathrm{AB} 07$ & -6.8 & -6.4 & -6.6 & -0.4 & -0.2 \\
\hline & AB08 & -4.0 & -7.4 & -4.8 & $3.4^{\mathrm{b}}$ & $2.6^{\mathrm{a}}$ \\
\hline & GROUP & -7.4 & -7.1 & -6.0 & -0.3 & $1.1^{\mathrm{a}}$ \\
\hline \multirow[t]{9}{*}{ Experiment 2: $2 \mathrm{FBs}, f_{\mathrm{m}}=8 \mathrm{~Hz}$, acoustic envelopes } & AB01 & -9.4 & -7.8 & -7.4 & -1.6 & 0.4 \\
\hline & $\mathrm{ABO} 2$ & -10.1 & -9.1 & -5.9 & -1.0 & $3.2^{\mathrm{a}}$ \\
\hline & AB03 & -6.8 & -6.2 & -4.5 & -0.6 & 1.7 \\
\hline & ABO4 & -7.3 & -6.3 & -4.2 & -1.0 & 2.0 \\
\hline & AB05 & -12.2 & -10.5 & -7.6 & $-1.8^{\mathrm{a}}$ & $2.9^{\mathrm{a}}$ \\
\hline & AB06 & -8.5 & -9.2 & 1.3 & 0.7 & $10.5^{\mathrm{a}}$ \\
\hline & $\mathrm{AB} 07$ & -9.6 & -6.1 & -2.0 & $-3.5^{\mathrm{a}}$ & $4.1^{\mathrm{a}}$ \\
\hline & AB08 & -8.4 & -7.5 & -5.6 & -0.9 & $1.9^{\mathrm{a}}$ \\
\hline & GROUP & -8.8 & -7.6 & -4.3 & $-1.2^{\mathrm{a}}$ & $3.3^{\mathrm{a}}$ \\
\hline \multirow[t]{8}{*}{ Experiment 3: $2 \mathrm{FBs}, f_{\mathrm{m}}=8 \mathrm{~Hz}$, deterministic envelopes } & AB02 & -3.0 & -2.8 & -2.6 & -0.2 & 0.2 \\
\hline & AB03 & -3.7 & -4.0 & -2.8 & 0.3 & $1.2^{\mathrm{a}}$ \\
\hline & $\mathrm{AB} 04$ & -2.9 & -2.8 & -2.3 & -0.1 & $0.6^{\mathrm{a}}$ \\
\hline & AB05 & -6.4 & -5.4 & -4.1 & $-1.0^{\mathrm{a}}$ & $1.3^{\mathrm{a}}$ \\
\hline & $\mathrm{AB} 06$ & -3.4 & -2.9 & -2.2 & -0.5 & $0.7^{\mathrm{a}}$ \\
\hline & $\mathrm{AB} 07$ & -3.5 & -2.8 & -1.5 & $-0.7^{\mathrm{a}}$ & $1.2^{\mathrm{a}}$ \\
\hline & AB08 & -6.2 & -3.9 & -1.4 & $-2.3^{\mathrm{a}}$ & $2.5^{\mathrm{a}}$ \\
\hline & GROUP & -4.1 & -3.5 & -2.4 & $-0.6^{\mathrm{a}}$ & $1.1^{\mathrm{a}}$ \\
\hline \multirow[t]{9}{*}{ Experiment $4: 1 \mathrm{FB}, f_{\mathrm{m}}=8 \mathrm{~Hz}$, acoustic envelopes } & AB01 & -11.6 & -12.0 & -8.4 & 0.3 & $3.6^{\mathrm{a}}$ \\
\hline & $\mathrm{ABO} 2$ & -7.6 & -8.4 & -5.7 & 0.8 & $2.6^{\mathrm{a}}$ \\
\hline & ABO3 & -4.2 & -6.3 & -2.3 & 2.1 & $4.0^{\mathrm{a}}$ \\
\hline & $\mathrm{ABO} 4$ & -7.1 & -7.0 & -6.5 & -0.1 & 0.5 \\
\hline & AB05 & -10.2 & -10.3 & -4.8 & 0.2 & $5.5^{\mathrm{a}}$ \\
\hline & AB06 & -5.2 & -3.3 & -2.2 & -1.9 & 1.2 \\
\hline & AB07 & -10.4 & -10.0 & -0.7 & -0.4 & $9.3^{\mathrm{a}}$ \\
\hline & AB08 & -6.2 & -10.7 & -8.0 & $4.6^{b}$ & $2.7^{\mathrm{a}}$ \\
\hline & GROUP & -7.6 & -8.3 & -4.5 & 0.7 & $3.8^{\mathrm{a}}$ \\
\hline
\end{tabular}

Note that positive values indicate a benefit from comodulation, i.e. CMR

${ }^{\text {a }}$ Significant at alpha $=0.05$

${ }^{\mathrm{b}}$ Significant at alpha $=0.006$

the OFB masker. This was done to test if a lower modulation rate, and hence longer dips in the OFB masker, would improve CMR in CI users.

\section{Experiment 2: Reducing SAM Rate}

Thresholds for $8 \mathrm{~Hz}$ modulation rate are plotted in the right column of Figure 3. The group SNR in the OFBonly condition was about $-8.8 \mathrm{~dB}$ and thus somewhat lower than in experiment $1(-7.4 \mathrm{~dB})$, indicating some release from forward masking due to reducing the SAM rate. Adding the $\mathrm{CM}$ FBs did not improve detection (threshold -7.6 dB) and hence CMR1 was not significant on an individual nor on a group level. More pronounced effects of the lower rate were seen for CMR2 which was significant in five of the eight listeners (AB02 and $\mathrm{AB} 05-\mathrm{AB} 08$ ). On a group level, CMR2 increased to
$3.3 \mathrm{~dB}$ and remained significant. The increase of CMR2 was not only driven by lower CM thresholds, but also by increased thresholds in the AntiM condition $(-4.3 \mathrm{~dB})$. The latter indicates that at the lower modulation rate, comodulation of all masker envelopes can give a larger release from the increased masking on the OFB electrode caused by the anti-modulated FBs.

Lowering the modulation rate in experiment 2 was done to aid listening in the masker dips and led to somewhat increased CMR2, but not CMR1. Changes in the maxima of the OFB-plus-signal envelope due to the addition of the tonal signal to the OFB noise could potentially be used by CI users as a detection cue (Moore et al. 1990). Implant users can take advantage of very small loudness cues to detect peak-to-peak variations of a signal (McKay and Henshall 2010). If listeners 
detected the signal in the modulation peaks rather than in the dips, it might have prevented CMR1.

Furthermore, Eddins and Wright (1994) observed more than $10 \mathrm{~dB}$ larger CMR when the OFB and FBs were $10 \mathrm{~Hz}$ SAM tones rather than narrow-band noises with a similar envelope fluctuation rate (bandwidth of $16 \mathrm{~Hz}$ ). This result implies that controlling the randomness in the envelopes by using deterministic envelopes is advantageous for CMR.

In experiments 1 and 2, we used envelopes of amplitude-modulated random noises for the OFB and FB pulse trains. This introduced random peaks from intrinsic instantaneous level fluctuations in the noise carriers into the SAM. These fluctuations could have worsened detection within the dips of the OFB masker and CMR or might have led to confusing detection cues due to changes of the peaks in the OFB envelope. In the following experiment, we tested the effect of reducing envelope variance on CMR by using fully deterministic envelopes.

\section{Experiment 3: Controlling Envelope Fluctuations}

The results of experiment 3 are shown in Figure 4 as SNRs of the signal threshold currents relative to the current of the target pulse train $(\mathrm{Ib})$ before modulation. Introducing deterministic envelopes did not improve CMR1. CMR1 was neither apparent on an individual nor on group level since group SNRs increased only slightly from -4.1 to $-3.5 \mathrm{~dB}$ when adding co-modulated FBs to the OFB. However, CMR2 was robust also with deterministic envelopes, and it was significant in six of the seven participants (AB03-AB08). On the group level, CMR2 was $1.1 \mathrm{~dB}$ and significant (-3.5 dB SNR for CM FBs vs -2.4 dB SNR with AntiM FBs). Note that here SNR directly relates to electric current, whereas in experiments 1, 2, and $4 \mathrm{SNR}$ was calculated from acoustic stimuli prior to the compressive current mapping. Considering that the electric dynamic range is three to four times smaller than the $30 \mathrm{~dB}$ of mapped acoustic range, the CMR2 magnitude of $1.1 \mathrm{~dB}$ in experiment 3 can be considered equal to that in experiment 2 with the same SAM (3.3 dB).

The strong prevalence of CMR2 in all previous experiments might be due to masking caused by current spread from the FB electrodes to the OFB electrode site. Current spread will be largest during the modulation maxima in the FBs. Thus, maximum masking in the AntiM condition occurs when the probe detection can be the best, i.e., during the OFB modulation minima. The finding that thresholds were higher in the AntiM than in the OFB-only condition is consistent with this idea, though other mechanisms, such as modulation detection interference, could have also contributed (Chatterjee 2003; Chatterjee and Oba 2004). We conducted a supplementary experiment to measure masking from the OFB to the FB electrodes (data not shown). Although masking declined over the (typically) four-electrode separation, the masked thresholds did not always reach the threshold in quiet suggesting spread of current across selected channels. This is consistent with the masking patterns found in other CI studies (Chatterjee and Shannon 1998).

In experiment 4, we investigated whether reducing current interactions in the OFB channel would improve CMR. Current summation was reduced by reducing the number of stimulated FB channels to one FB electrode. Electrode separation could therefore be increased to nine electrodes, about $7.65 \mathrm{~mm}$ distance between the contacts of the Advanced Bionics' HiFocus ${ }^{\circledR}$ Helix electrode array (Advanced Bionics 2008), to reduce current spread to the OFB channel. As in experiments 1 and 2, experiment 4 used acoustic envelopes because they convey cues from the beating of the noise and the

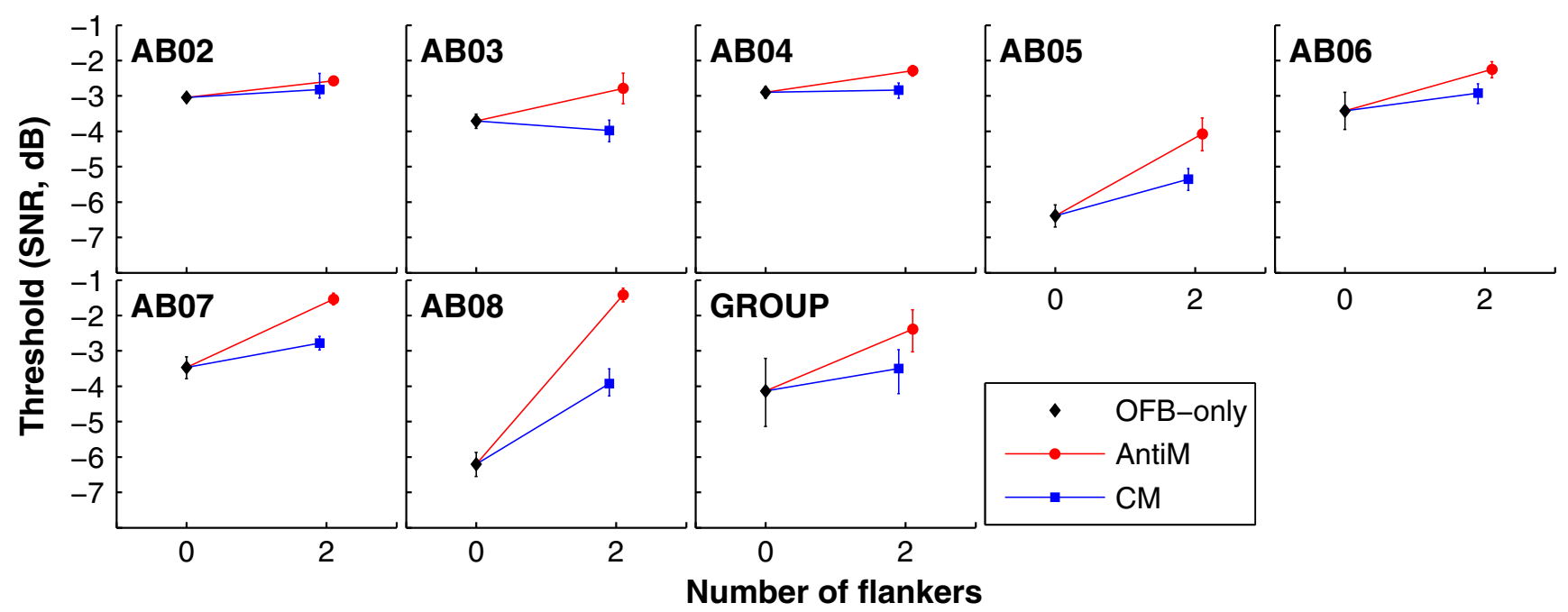

FIG. 4. Signal thresholds in the OFB-only (diamonds), co-modulated (CM, squares) and anti-modulated (AntiM, circles) conditions. The SAM of $8 \mathrm{~Hz}$ was applied directly to electric pulse trains. Signal thresholds are presented as in Fig. 3. 


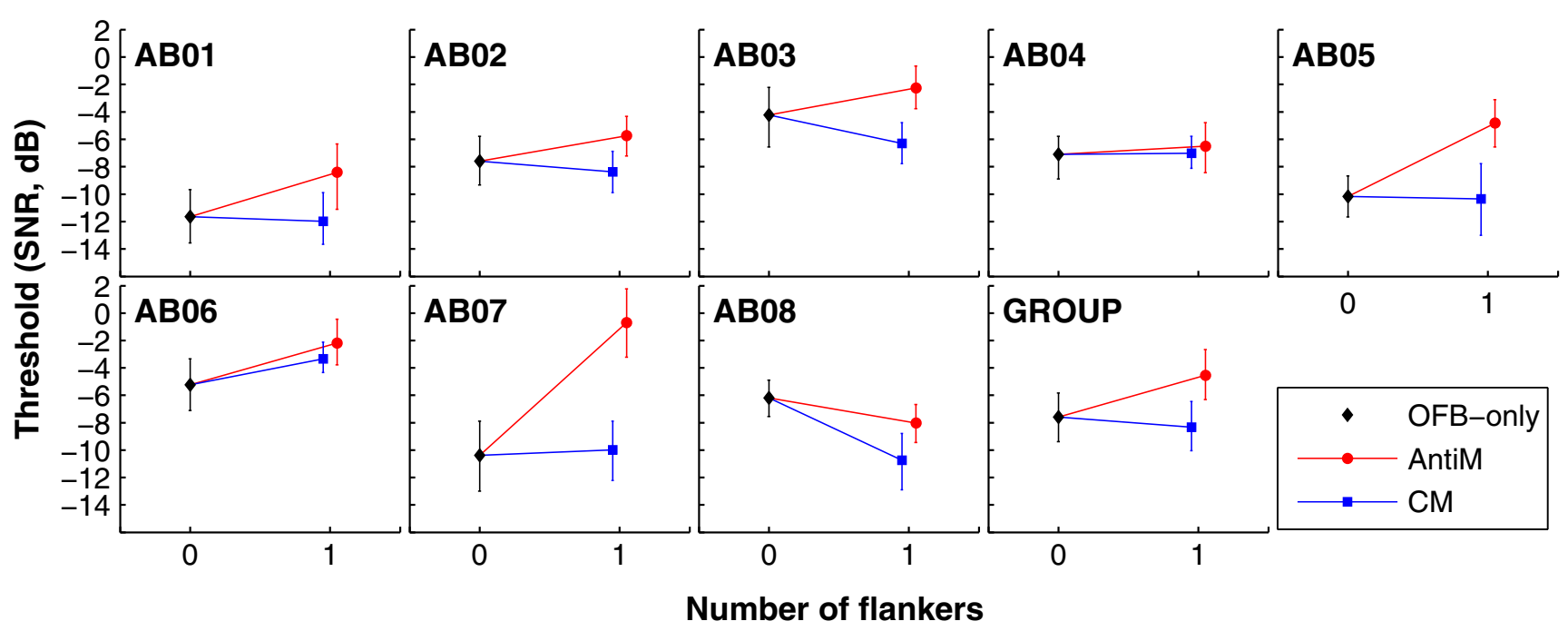

FIG. 5. Signal thresholds of experiment 4 with a single FB presented at a 9-electrode separation from the OFB electrode. The symbols show OFB-only (diamonds), co-modulated (CM, squares) and anti-modulated (AntiM, circles) conditions, respec-

signal within the OFB channel and might have improved CMR1 in those experiments. To mimic the fully correlated noise envelopes of experiment 3 , we used the OFB envelope also on the FB electrode in the CM condition.

\section{Experiment 4: Increasing Electrode Separations}

Figure 5 shows the thresholds measured in experiment 4 with the OFB and a single FB spaced nine electrodes apart. The across-participant average SNR for the OFB alone was $-7.6 \mathrm{~dB}$, similar to the previous experiments with acoustic stimuli, despite using a different OFB electrode. Interestingly, average thresholds in the CM condition were lower than in the OFBonly condition $(-8.3 \mathrm{~dB})$, indicating a trend for CMR1. However, CMR1 was not significant on a group level. Analysis of individual data showed a large CMR1 of $4.3 \mathrm{~dB}$ for participant AB08, and it remained significant after Bonferroni correction. A trend for CMR1, though not reaching significance, was also found for participants AB01-AB03 and AB05. The increased prevalence of CMR1 in experiment 4 indicates that for wider electrode separation and for high envelope coherence adding co-modulated flanking bands can reduce detection thresholds below those found on a single electrode and thus support CMR1.

CMR2 was significant for all participants except AB04 and AB06. It was again significant on a group level. Its magnitude was somewhat larger than in previous experiments because CM thresholds were lower, but AntiM thresholds remained about the same (c.f. $-4.3 \mathrm{~dB}$ vs $-4.5 \mathrm{~dB}$ in experiments 2 and 4 , respectively, where the same stimuli were used). The latter indicates that increasing the electrode separa- tively. The co-modulated condition had maximum envelope correlations, i.e., $\mathrm{FB}=\mathrm{OFB}(r=1)$. The $\mathrm{SAM}$ rate was $8 \mathrm{~Hz}$. Thresholds are presented as in Fig. 3.

tion and reducing the number of FBs either did not result in reduced current spread into the OFB channel, or that other mechanisms determined probe thresholds in the AntiM condition.

\section{DISCUSSION}

\section{CMR in Electric Hearing}

The present study tested CMR in electric hearing with eight users of CIs by stimulating their auditory nerve with modulated electric pulse trains via a research interface. Two facets of CMR were examined. Following the strict definition of CMR, we investigated if adding co-modulated FBs to a modulated OFB masker lowered detection thresholds of a probe presented with the OFB masker. In this CMR1 condition, the effect did not reach significance on a group level, but one of the eight participants exhibited CMR in several experiments after Bonferroni correction indicating a robust effect. Stimuli were manipulated in numerous ways to promote CMR1. CMR1 was strongest in experiment 4 (significant in one participant and a further four showing a clear trend) for a large electrode separation to reduce current spread and for acoustic envelopes that were coherent in FB and OFB channels. We conclude that CMR1 is generally a weak effect, but can be available and robust in selected participants.

The second facet, CMR2, was observed in each experiment and in most participants, i.e., co-modulated pulse trains on all electrodes produced lower thresholds than anti-modulated pulse trains. Furthermore, decreasing modulation rate, reducing envelope 
variance, and increasing OFB-FB separation increased the prevalence of CMR2 across participants. This result leads us to conclude that comodulation can improve signal detection in electric hearing, at least in specific conditions. Since CMR2-type benefits were shown for comodulation across multiple electrodes, they may be relevant in realistic situations.

Several factors could have weakened CMR1 in our experiments. Experiment 1 used identical envelopes as in our previous study with $\mathrm{NH}$ listeners, where the CI processing was simulated by vocoding stimuli (Pierzycki and Seeber 2010). CMR1 was observed despite vocoding, but its magnitude was reduced compared to a condition with unprocessed stimuli. The vocoding replaces the temporal fine structure of the signals with noise which might have been one reason for the reduced CMR1. Similarly, in the present study fine timing cues were not coded in the electric pulses delivered to the CI which might have reduced CMR. Adding co-modulated FBs lowered thresholds more consistently only in experiment 4 with wide OFB-FB separation. This suggests that current spread might have been a factor in reducing CMR1. Furthermore, a finer frequency resolution than that provided by a CI and intact cochlear processes such as compression or suppression could have provided the additional cues for the NH listeners and led to CMR with vocoded stimuli.

The temporal envelopes of pulse trains varied at a slow rate due to the applied SAM and at faster rates due to modulations of the random noise carriers. This led to level fluctuations in the envelope peaks that would change forward masking in every trial, affecting the detection of the signal in the masker dips. However, neither reducing the SAM rate to $8 \mathrm{~Hz}$ to temporally extend the dips in the masker (experiment 2), nor using ideal sinusoidal envelopes without random level fluctuations (experiment 3 ) significantly reduced CM thresholds to improve CMR1.

CMR2 was consistently observed in all experiments in general agreement with recent findings by Zirn et al. (2013) who showed CMR2 in about a third of their CI users. We demonstrated a relationship with increased AntiM thresholds with respect to OFB-only thresholds. We attributed this masking increase to current spread from the FBs into the OFB channel for experiments with a 4-electrode separation of the FBs (about $3.4 \mathrm{~mm}$ distance in the Advanced Bionics' HiFocus ${ }^{\circledR}$ Helix electrode array; Advanced Bionics 2008). In experiment 4 we tested this hypothesis by increasing the OFB-FB separation to nine electrodes (about $7.65 \mathrm{~mm}$ distance). Interestingly, AntiM thresholds, which should be most affected by current spread, were unchanged (c.f. experiment 2 and 4 with the same stimuli, Table 2). Although we cannot exclude the possibility of masking across cochlear turns (Micco and Richter 2006), we assume that the large OFB-FB separation and the use of only a single FB reduced the current spread. The results therefore suggest that current spread was not the only factor affecting AntiM thresholds and CMR2. Mechanisms similar to modulation detection interference (MDI, see below) might have made it harder to detect the probe during the modulation maxima of the FBs (Chatterjee 2003; Chatterjee and Oba 2004).

This observation differs from NH where CMR is known to be smaller for large FB separations and for a smaller number of FBs (Schooneveldt and Moore 1987; Hall et al. 1990; Cohen 1991; Ernst and Verhey 2005). One reason for the strong prevalence of CMR2 with the large FB separation might be an increased independence of the OFB and FB channels (Moore et al. 1993). This is also supported by the fact that comodulated thresholds were generally lower than the OFB-only thresholds in experiment 4. Channel independence could be further increased with current focusing by using bipolar or tripolar rather than monopolar stimulation. Although stimulation in bipolar or tripolar mode might not be optimal for all cochlear implant users because it requires larger currents and has been shown to cause inconsistent changes in excitation patterns between the active and return electrodes (Kwon and van den Honert 2006), it might provide some benefit for CMR in users who show narrower spread of excitation due to current focusing (Landsberger et al. 2012).

Another explanation for CMR2 could be an interference caused by the FB maximum in detecting the change caused by the probe in the OFB channel, a process related to MDI. MDI was evidenced by impoverished detection of changes to the modulation depth in a target channel when a modulated masker is presented in a remote channel (Chatterjee 2003; Chatterjee and Oba 2004). Here, the addition of the anti-modulated FBs could worsen the detection of the decrease in modulation depth in the OFB channel due to the signal filling in the masker's dips. This could also explain high AntiM thresholds in all experiments. Chatterjee and Oba (2004) observed MDI for electrode separations as large as $11 \mathrm{~mm}$ which is wider than the separation between OFB and FB electrodes in experiment 4. Thus, CMR2 observed in our experiments may also be connected with a "release" from MDI.

\section{Consistency with CMR Mechanisms and Models}

The CMR is often attributed to a comparison of dips in the OFB and FBs as a cue for when to listen for the signal in the OFB channel-the dip-listening model (Buus 1985). Detection and temporal processing of gaps in CI users appears similar to that in NH listeners (Grose and 
Buss 2007). In spite of this, CI users show only a limited release from masking in speech perception in modulated maskers (Stickney et al. 2004) and relatively long gap durations in a masker are needed to improve speech understanding in electric hearing (Nelson et al. 2003). To promote listening in the masker dips, we reduced the SAM rate to obtain longer dip durations. Furthermore, as reducing the envelope fluctuations can improve withinchannel gap detection in electric hearing (Grose and Buss 2007), we also used deterministic envelopes to control the envelope fluctuations. For both of these manipulations, we observed an increased prevalence of CMR2 across participants, though CMR1 was not observed.

The benefit from reducing envelope fluctuations appears to support the equalization-cancelation model of CMR. Increasing the similarity of envelopes in electric hearing could improve the equalization of the $\mathrm{OFB}$ and $\mathrm{FB}$ envelopes and provide appropriate input for the cancelation stage of the model. This would maximize the SNR in the OFB channel more effectively and improve signal detection. The fact that CMR1 was obtained in experiment 4 with random but fully coherent envelopes supports this.

The present study tested CMR in electric hearing with direct stimulation technique, i.e. in the absence of compression or suppression effects of the cochlea. Significant CMR1 and 2 were found for individual participants suggesting that cochlear compression or suppression is not required for CMR.

The signal detection could have been impaired by a limited perceptual segregation between signal and masker caused by their similar pitches and by the absence of TFS cues in electric hearing. TFS cues were not explicitly encoded in the present study, but were shown to contribute to the magnitude of CMR in NH (Schooneveldt and Moore 1987; Pierzycki and Seeber 2010). Our CI participants nevertheless consistently reported hearing a clear "beep" in the presence of a "dialling tone" background, suggesting that the temporal properties of the signal and masker were discriminable. However, it seems that both signal and masker were perceived as having a tone-like pitch, presumably relating to the place pitch of the OFB electrode (Carlyon et al. 2010).

\section{Implications for Development and Performance with Cls}

Significant CMR2 found in all experiments suggests that comodulation can generally improve signal detection in noise in electric hearing. This may occur in situations where envelopes are highly co-modulated across channels or by applying specific envelope processing algorithms. These approaches would have to increase the across-electrode similarity of the low-rate envelope modulations that are faithfully represented in the auditory nerve and at higher levels of the auditory system (Middlebrooks 2008). The recently proposed modified F0mod strategy, in which the sound's fundamental frequency (F0) is estimated and used for the SAM of the envelopes of all CI processor output channels, is an example of a strategy that provides comodulation across electrodes (Milczynski et al. 2009). However, the present results demonstrate that obtaining consistent CMR2 requires sufficient channel independence-a condition that is difficult to achieve with current CIs. Furthermore, the results of our previous studies in NH with vocoded stimuli (Pierzycki and Seeber 2010) suggest that improved encoding of the TFS information would be needed to provide additional cues for segregation of the signal from the masker. These limitations would add to the lack of benefit from comodulation and CMR for speech perception in noise in CI users (Ihlefeld et al. 2012).

\section{ACKNOWLEDGMENTS}

We would like to thank the participants for taking part in our experiments, for their time and efforts. We thank Gerard O'Donoghue, Tracey Twomey, and Ellen Jeffs of the Nottingham Auditory Implant Programme for help with participant recruitment. We also thank Patrick Boyle and Waldo Nogueira of the Advanced Bionics Corporation for providing the direct stimulation hardware and software, Mark Edmondson-Jones for help with statistical analysis, and Jesko Verhey for helpful discussions. We are grateful to Bob Carlyon, three anonymous Reviewers and the Editor-inChief for helpful comments and suggestions.

Open Access This article is distributed under the terms of the Creative Commons Attribution License which permits any use, distribution, and reproduction in any medium, provided the original author(s) and the source are credited.

\section{REFERENCES}

Advanced Bionics (2008) HiFocus ${ }^{\circledR}$ electrode series, pp 35-62

Buus S (1985) Release from masking caused by envelope fluctuations. J Acoust Soc Am 78:1958-1965

Carlyon RP, Geurts L, Wouters J (2000) Detection of small acrosschannel timing differences by cochlear implantees. Hear Res 141:140-154

Carlyon RP, Deeks JM, McKay CM (2010) The upper limit of temporal pitch for cochlear-implant listeners: stimulus duration, conditioner pulses, and the number of electrodes stimulated. J Acoust Soc Am 127:1469-1478

Chatterjee M (2003) Modulation masking in cochlear implant listeners: envelope versus tonotopic components. J Acoust Soc Am 113:2042-2053

Chatterjee M, Shannon RV (1998) Forward masked excitation patterns in multielectrode electrical stimulation. J Acoust Soc Am 103:2565-2572

Chatterjee M, Ова SI (2004) Across- and within-channel envelope interactions in cochlear implant listeners. J Assoc Res Otolaryngol 5:360-375 
Cohen MF (1991) Comodulation masking release over a three octave range. J Acoust Soc Am 90:1381-1384

EDdins DA, WRIGHT BA (1994) Comodulation masking release for single and multiple rates of envelope fluctuation. J Acoust Soc Am 96:3432-3442

Efron B, Tibshirani RJ (1993) An introduction to the bootstrap. Chapman \& Hall, New York

ERnst SMA, Verhey JL (2005) Comodulation masking release over a three octave range. Acta Acustica United Acustica 91:998-1006

Ernst SMA, Rennies J, Kollmeier B, Verhey JL (2010) Suppression and comodulation masking release in normal-hearing and hearing-impaired listeners. J Acoust Soc Am 128:300-309

Goldman SA, Baer T, Moore BCJ (2010) Within-channel cues to comodulation masking release for single and symmetrically placed pairs of flanking bands. J Acoust Soc Am 128:29882997

Grose JH, Buss E (2007) Within- and across-channel gap detection in cochlear implant listeners. J Acoust Soc Am 122:3651-3658

Hall JW, Haggard MP, Fernandes MA (1984) Detection in noise by spectro-temporal pattern analysis. J Acoust Soc Am 76:50-56

Hall JW, Grose JH, HagGard MP (1990) Effects of flanking band proximity, number, and modulation pattern on comodulation masking release. J Acoust Soc Am 87:269-283

Ihlefeld A, Shinn-Cunningham BG, Carlyon RP (2012) Comodulation masking release in speech identification with real and simulated cochlear-implant hearing. J Acoust Soc Am 131:1315-1324

KWON BJ, van DEN HonerT C (2006) Effect of electrode configuration on psychophysical forward masking in cochlear implant listeners. J Acoust Soc Am 119:2994-3002

Landsberger DM, Padilla M, Srinivasan AG (2012) Reducing current spread using current focusing in cochlear implant users. Hear Res 284:16-24

LEVITT H (1971) Transformed up-down methods in psychoacoustics. J Acoust Soc Am 49:467-477

McFadden D (1986) Comodulation masking release: Effects of varying the level, duration, and time delay of the cue band. J Acoust Soc Am 80:1658-1667

McKay C, Henshall K (2010) Amplitude modulation and loudness in cochlear implantees. J Assoc Res Otolaryngol 11:101-111

Micco AG, Richter C-P (2006) Tissue resistivities determine the current flow in the cochlea. Curr Opin Otolaryngol Head Neck Surg 14:352-355
Middlebrooks JC (2008) Auditory cortex phase locking to amplitudemodulated cochlear implant pulse trains. J Neurophysiol 100:76-91

Milczynski M, Wouters J, van Wieringen A (2009) Improved fundamental frequency coding in cochlear implant signal processing. J Acoust Soc Am 125:2260-2271

Moore BCJ, Glasberg BR, Schooneveldt GP (1990) Across-channel masking and comodulation masking release. J Acoust Soc Am 87:1683-1694

Moore BCJ, Shailer MJ, Hall JW III, Schooneveldt GP (1993) Comodulation masking release in subjects with unilateral and bilateral hearing impairment. J Acoust Soc Am 93:435-451

Nelson PB, Jin S-H, Carney AE, Nelson DA (2003) Understanding speech in modulated interference: Cochlear implant users and normal-hearing listeners. J Acoust Soc Am 113:961-968

Pierzycki RH, Seeber BU (2010) Indications for temporal fine structure contribution to co-modulation masking release. J Acoust Soc Am 128:3614-3624

RichaRDS VM (1987) Monaural envelope correlation perception. J Acoust Soc Am 82:1621-1630

Schooneveldt GP, Moore BCJ (1987) Comodulation masking release (CMR): Effects of signal frequency, flanking-band frequency, masker bandwidth, flanking-band level, and monotic versus dichotic presentation of the flanking band. J Acoust Soc Am 82:1944-1956

Stickney GS, Zeng F-G, Litovsky R, Assmann P (2004) Cochlear implant speech recognition with speech maskers. J Acoust Soc Am 116:1081-1091

Verhey JL, Dau T, Kollmeier B (1999) Within-channel cues in comodulation masking release (CMR): Experiments and model predictions using a modulation-filterbank model. J Acoust Soc Am 106:2733-2745

Verhey JL, Pressnitzer D, Winter IM (2003) The psychophysics and physiology of comodulation masking release. Exp Brain Res 153:405-417

WAGNER E (2002) Across-channel processing in auditory perception. Doctoral Thesis, Technical University München

Wilson BS, Finley CC, Lawson DT, Wolford RD, Eddington DK, RABINOWITZ WM (1991) Better speech recognition with cochlear implants. Nature 352:236-238

Zirn S, Hempel JM, Schuster M, Hemmert W (2013) Comodulation Masking Release induced by controlled electrical stimulation of auditory nerve fibers. Hear Res 296:60-66 